\title{
Neurolinguistisches Programmieren (NLP) im Gründungsmanagement
}

\author{
Prof. Dr. rer. oec. Willi K. M. Dieterle
}

\section{Problemstellung}

Nicht selten werden von Unternehmensberatern gescheiterte Gründungsprojekte geschildert, die trotz bester formaler Voraussetzungen - hinsichtlich Rentabilitätsentwicklung, Liquiditätsverlauf etc. - relativ unerwartet versanden. Eine nähere Analyse zeigt dann jedoch, daß relativ häufig defizitäre Kommunikationsprozesse diese Entwicklung maßgeblich beeinflußt haben. Dies gilt sowohl für externe Prozesse, wie fehlgeschlagene Aquisition von Aufträgen, als auch für interne Prozesse, wie innere Dialoge des Gründers, beispielsweise Selbstzweifel hinsichtlich der Tragfähigkeit des Projektes.

Vor diesem Hintergrund stellt sich die Frage, wie derartige Kommunikationsprozesse effektiver gestaltet werden können, um einen nachhaltigen Gründungserfolg zu gewährleisten.

In Management-Kommunikationstrainings wird hierbei immer häufiger mit der Methodik des Neurolinguistischen-Programmierens (NLP) gearbeitet. Welche Lösungsbeiträge bzw. Hilfestellungen dieser Ansatz in dem hier interessierenden Problemfeld des Gründungsmanagement generieren kann, ist bislang jedoch noch nicht untersucht worden.

\section{Begriffliches und theoretische Fundierung}

Das NLP ist Anfang der siebziger Jahre in den USA von dem DV-Experten Richard Bandler und dem Linguist John Grinder entwickelt worden, die hierbei die grundlegende Frage stellten: Wie kommunizieren erfolgreiche Kommunikatoren? Mit Hilfe der Methode des Master Modeling wurden Verhaltensmuster erfolgreicher Kommunikatoren beobachtet, analysiert und systematisiert; das in diesem Zusammenhang entstehende MasterModell repräsentiert die relevanten Erfolgsparameter der modellierten Persönlichkeit.

Eine grundlegende Fundierung des NLP-Ansatzes ist insbesondere Robert Dilts ${ }^{1}$ gelungen, der auf Erkenntnisse der Kybernetiker, der Systemdenker und der Neurophysiologen rekurriert. Die dynamische Entwicklung dieses Ansatzes hat schließlich dazu geführt, daß während der letzten Jahre eine Reihe differenzierter NLPModelle insbesondere in den Bereichen Management, Gesundheit und Therapie entwickelt worden sind.

Zur Begrifflichkeit:

Neuro beinhaltet die unterschiedlichen Zustände des senso-motorischen Nervensystems.

Linguistisch impliziert die modellhafte Darstellung neuronaler Prozesse durch Sprache.
Programmieren bezieht sich auf die Organisation derartiger Prozesse.

Aufgrund dieser neurolinguistischen Fundierung erheben Vertreter des NLP-Ansatzes den Anspruch einer Wissenschaft der subjektiven Erfahrung.

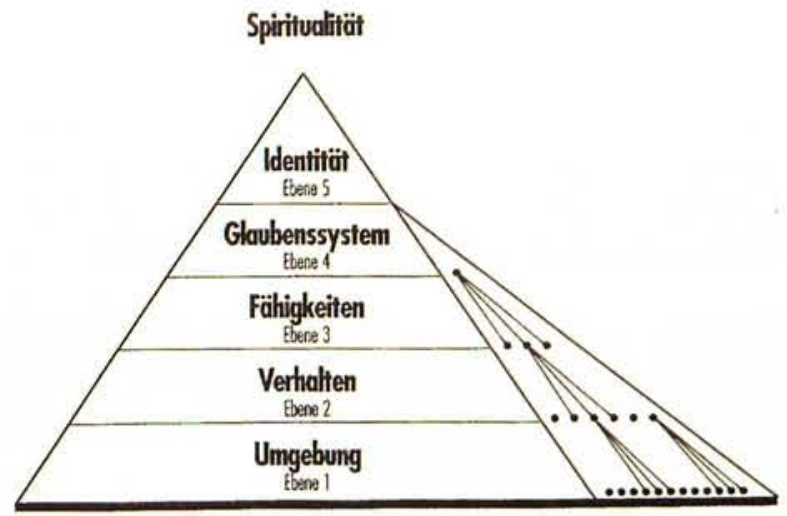

Abb. 1: Logische Ebenen (NLP)

\section{Interventionsebenen}

Die Leistungsfähigkeit des NLP-Ansatzes wird vor allem mit einer Mehr-Ebenen-Analyse begründet, die zum Ausweis sog. logischer Ebenen ${ }^{2}$ führt (Abb. 1). Hierbei umfaßt die unterste Ebene Umgebung bestimmte Verhaltensweisen in bestimmten Situationen, zu bestimmten Zeitpunkten, an bestimmten Orten etc.. Diese Verhaltensweisen können in Abhängigkeit von unterschiedlichen Kontextfaktoren (Rahmenbedingungen) mehr oder weniger stark modifiziert werden. Werden auf der zweiten Ebene neue Verhaltensmuster entwikkelt, führt dies auf der anderen Seite zu veränderten Situationsspezifika. Das Verhaltensspektrum ist wiederum von den jeweils entwickelten Fähigkeiten abhängig; so kann beispielsweise eine Fähigkeit wie Selbstvertrauen eine entscheidende Voraussetzung für den Gründungsentschluß sein. Noch bedeutsamer ist aus NLPSicht jedoch die vierte Ebene der Werte, Normen und Glaubenssätze. Sie determiniert das menschliche Handeln schlechthin und damit die jeweiligen Ausprägungsformen auf den darunter liegenden Ebenen. Begrenzende, einengende Glaubenssysteme im Sinne eines ich kann nicht sind mit hoher Wahrscheinlichkeit ein KOKriterium für das geplante Gründungsprojekt.

Unterstïtzend können in diesem Zusammenhang mentale NLP-Methoden wie der New Belief Generator eingesetzt werden, die relativ kurzfristig die gewünschten Erfolge herbeifuihren. Bei der Auflösung einschränkender bzw. der Generierung neuer Glaubenssysteme werden Interventionen empfohlen, die auf einer Meta-Ebene jenseits des Problemkontextes liegen. 


\section{Ressource Strategien}

Auf dieser Ebene der Veränderung stellt sich zunächst die Frage nach dem individuellen Leistungsspektrum und den Leistungspotentialen, die in die unternehmerische Existenz eingebracht werden können. Bei der Analyse dieser persönlichen Ressourcen sollten alle relevanten Erfahrungsebenen in den Analyseprozeß integriert werden. Hierbei wird im Sinne des NLP-Ansatzes von folgender Dreigliederung ausgegangen:

$$
\begin{aligned}
& \text { Spiritualität - Erkennen } \\
& \text { Bewußtsein - } \\
& \text { Körper - Wahrnehmen }
\end{aligned}
$$

Dieses Analyseraster führt zu folgenden möglichen Vorgehensweisen:

\section{- Erkennen}

Hierzu gehören intuitive Fähigkeiten - basierend auf transpersonalen Erfahrungen - die in den unternehmerischen Entwicklungsprozeß eingebracht werden können.

\section{- Denken / Fühlen}

Auf dieser Ebene sind sowohl fachspezifische bzw. fachübergreifende Kenntnisse als auch prozessuale Aspekte zu reflektieren. Zu letzteren gehören insbesondere im linkshemisphärischen Bereich des Gehirns logisch-systematische Analysefähigkeiten, im rechtshemisphärischen Bereich kreativitätsrelevante Fähigkeiten wie einfallsreiches, innovatives und vernetztes Denken. Darüber hinaus Durchsetzungsfähigkeiten wie Überzeugungskraft, Beharrlichkeit, Entscheidungs- und Konfliktfähigkeit, aber auch Teamkompetenzen wie Kontakt-, Kooperations- und Integrationsfähigkeit.

Unterstützend kann auf diesen Ebenen die NLP Ressource Strategie eingesetzt werden.

Sie ermöglicht durch die Technik der Dissoziation (Übernahme der Rolle des Beobachters) ressourcevolle Positionen aufzubauen, die in künftigen Situationen zur Verfügung stehen.

\section{- Wahrnehmen}

Auf dieser Ebene sind sowohl die präferierten Wahrnehmungskanäle (visuell, auditiv etc.) als auch die bevorzugten Wahrnehmungsfelder (Technik, Natur etc.) von Bedeutung.

Hier empfiehlt sich die NLP-Analyse der Sinnesmodalitäten. Durch eine Veränderung dieser Modalitäten (Wahrnehmungsspezifika) können die damit im Zusammenhang stehenden Erfahrungen modifiziert und intensiviert werden. Neue internale Repräsentationen führen in der Folge zu ressourcevolleren $\mathrm{Zu}$ ständen ${ }^{4}$.

In einem zweiten Schritt stellt sich die Frage nach den Interessen und Bedürfnissen des Gründers. In diesem Zusammenhang hat der Gründer die Möglichkeit, das Spektrum und die Intensität seiner persönlichen Bedürfnisse näher kennenzulernen. Darüber hinaus werden grundlegende Wege der Bedürfnisbefriedigung aufgezeigt.

Hierbei kann eine Sensibilisierung für grundlegende

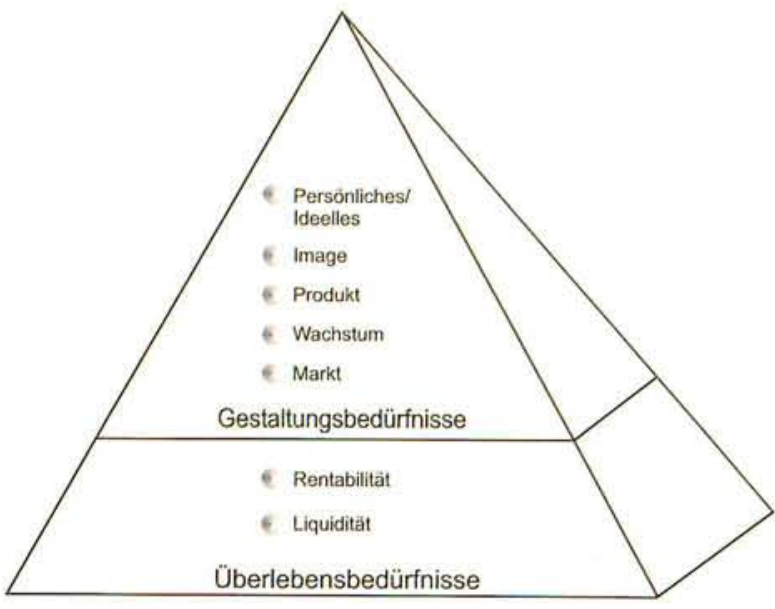

Abb. 2: Motivationale Ebenen

Möglichkeiten der Bedürfnisbefriedigung, die in einem Gründungsprojekt angelegt sind, erreicht werden. Ein weiteres Ziel liegt in der Generierung transparenter Strukturen und Prioritäten hinsichtlich der jeweiligen Beduirfnisse und Erwartungen.

Hilfreich ist in diesem Prozeß die NLP-Methodik der sensorischen Analyse. Durch eine Aktivierung insbesondere auditiver und visueller Wahrnehmungsfähigkeiten können kommunizierte Inkongruenzen identifiziert werden. Ihre erfolgreiche Bearbeitung führt zu einer verbesserten Handlungskompetenz ${ }^{5}$.

Auf der Basis der personalen Analyse stellt sich nunmehr die Frage nach erfolgversprechenden Geschäftsfeldern, in denen das Gründungsprojekt angesiedelt werden könnte. Um einen langfristigen Erfolg zu gewährleisten, empfiehlt sich hierbei die Reflektion sog. Meta-Trends, die Trendforscher „als die eigentlichen Bezugsgrößen für ein zukunftsgerichtetes Management"6 bezeichnen. Hierzu gehören zunehmende Individualisierung, Regionalisierung, Technisierung u.a. Eine fundierte Trendanalyse ist somit unabdingbar, um das Gründungsprojekt in attraktiven Märkten anzusiedeln und Marktnischen frühzeitig zu erkennen. Die in diesem Zusammenhang erforderliche Analysearbeit umfaßt in dieser Projektphase zunächst eine Sensibilisierung für aktuelle Trends mit dem Ziel, das persönliche Interesse des Gründers an der verantwortungsvollen Gestaltung wünschenswerter Zukünfte zu wecken.

Unterstützend kann hierbei mit der NLP Ressource Strategie gearbeitet werden. Sie fuihrt zu einer Aktivierung von Entwicklungpotentialen, um den zukünftigen Anforderungen gerecht zu werden. Darüber hinaus empfiehlt sich der Einsatz von NLP Interventionen im Bereich Werte / Überzeugungen.

\section{Visionsentwicklung}

Nachdem alle für den Unternehmensgründer relevanten Ressourcen in den Entwicklungsprozeß integriert worden sind, kann nunmehr die Generierung der Unter- 
nehmensvision ins Blickfeld gerüickt werden. Die zentrale Unternehmensidee oder Unternehmensvision ist der Leitgedanke bzw. das Leitbild, das im Sinne einer wünschenswerten Zukunft geschaffen werden soll. Als geeignete NLP-Intervention kommt die Walt Disney Strategie $^{7}$ in Frage, bei der sowohl analytische als auch intuitive Entwicklungsmomente berïicksichtigt werden.

Das Vorgehen vollzieht sich hierbei in drei Schritten:

1. Die Position des Träumers: Seine Aufgabe ist es, innerhalb eines spezifizierten Zielrahmens zu „träumen“, ohne sich darum zu kümmern, ob die jeweiligen Inhalte realistisch sind bzw. einer kritischen Überprïfung standhalten.

Als geeignete NLP-Methoden können hierbei Phantasiereisen, Metaphern, Rapport- und EntspannungsTechniken empfohlen werden.

2. Die Position des Realisten: In diesem Zusammenhang soll geklärt werden, ob die Träume Realisierungschancen haben.

Hier kann mit den NLP-Methoden Reframing, Ressource-Strategie und auf den Ebenen Identität / Werte gearbeitet werden.

3.Die Position des Kritikers: Hierbei soll konstruktive Kritik geleistet werden, indem Schwachstellen aufgezeigt und Wege zu deren Beseitigung entwickelt werden.

Methodisch hilfreich ist insbesondere der NLP Ökologie Check, um auch das weitere Umfeld des Grïnders (Familie, Freunde etc.) angemessen zu berüicksichtigen.

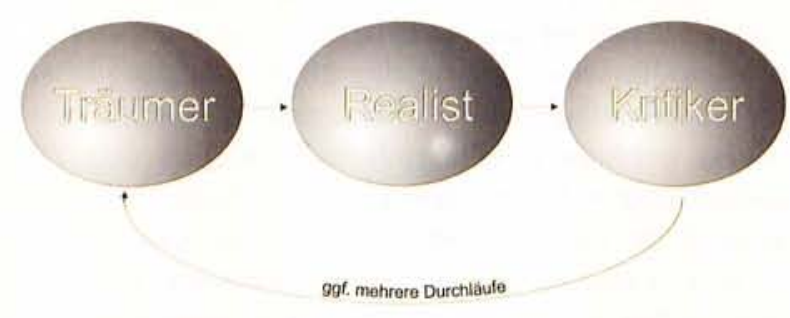

Abb. 3: Walt Disney Strategie

Der sich daran anschließende Entwicklungsschritt umfaßt die zunehmende Konkretisierung der Unternehmensvision. Dies beinhaltet einerseits eine zielgruppenorientierte Analyse der jeweiligen Anforderungen, beispielsweise auf Basis der o.g. Analyse der motivationalen Ebenen, andererseits eine weitergehende Reflektion seiner derzeit bestehenden Leistungs- bzw. MotivationsStrukturen. Im Rahmen dieser Vorgehensweise können dann möglicherweise vorhandene Entwicklungsperspektiven / -alternativen priorisiert werden.

Mit Hilfe der NLP Methoden der Ergebnisbestimmung (outcome frame) kann die Unternehmensvision im Sinne eines erwünschten Zustandes auf den sensorischen Ebenen des Unternehmensgründers verankert werden, d. h. bei der Ergebnisbestimmung müssen Evidenzen vorliegen im Sinne der Fragen: Wann, wo, wie, woran werden Sie feststellen, daß Sie Ihr Ergebnis erreicht haben? Welches Ergebnis, in welchem Kontext, mit welchen Personen etc.?

Unterstiitzend kann hierbei mit der Als-Ob-Technik gearbeitet werden $^{8}$, mit der die jeweiligen Ressourcen gefunden werden können, die erforderlich sind, um den erwünschten Zustand zu erreichen.

Falls limitierende Glaubensvorstellungen auf Seiten des Grüinders bestehen, die den Erfolg des Grüindungsvorhabens in Frage stellen könnten, empfiehlt es sich, diesen Begrenzungen positive Glaubenshaltungen entgegenzusetzen und sie auf einzelnen Wahrnehmungsebenen zu verankern (Veränderung der historischen Prägungen). NLP-methodisch läßt sich diese Vorgehensweise mit Hilfe sog. Submodalitäten realisieren. Daruiber hinaus ist den Faktoren innerhalb des Umfeldes, die beachtet und erhalten werden müssen, angemessen Rechnung zu tragen (Ökologie-Check).

\section{Entwicklung eines Geschäftsplanes}

Die unternehmerische Vision ist die Ausgangsbasis für die Entwicklung eines detaillierten Geschäftsplanes (business plans), der dem Gründungsunternehmer als Handlungsanleitung dient ${ }^{9}$. Sie erfüllt darüber hinaus Informationszwecke für potentielle Geschäftspartner, Banken etc. Ein Geschäftsplan könnte beispielsweise die in Abbildung 4 dargestellten Elemente beinhalten.

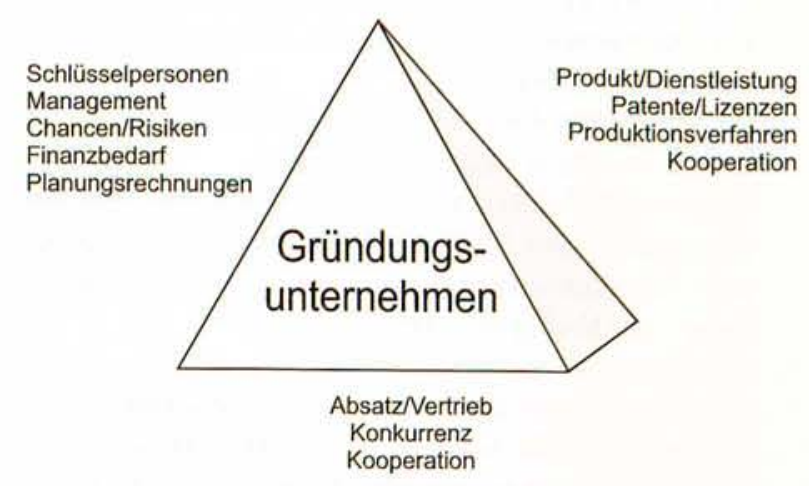

Abb. 4: Geschäftsplan (Business plan)

Bei der Konzeptualisierung eines Geschäftsplanes ist es von besonderer Bedeutung, die Erfolgsfaktoren des jeweiligen Gründungsprojektes herauszuarbeiten und Lösungswege für einzelne Problembereiche zu entwickeln.

Unterstützend kann hierbei insbesondere mit dem linguistisch orientierten Meta-Modell gearbeitet werden, das mit Hilfe von Fragetechniken die Möglichkeit bietet, die in der Oberflächenstruktur der Sprache bzw. des Textes enthaltenen Generalisierungen, Tilgungen und Verzerrungen zu hinterfragen, um eine effektivere Kommunikation zu gewährleisten. Damit können die Intentionen des Unternehmensgründers transparenter gemacht und die entsprechenden Aussagen präzisiert werden. 


\section{Fazif}

Die Entwicklung von Gründungsprojekten kann -wie im einzelnen gezeigt werden konnte -nicht nur auf betriebswirtschaftlich / technologischer Ebene, sondern auch durch den Einsatz geeigneter kommunikativer Methoden wirksam unterstiitzt werden. Dies setzt jedoch voraus, daß die NLP-Methodik situationsgerecht eingesetzt wird, d.h. an den jeweiligen Bedürfnissen / Erwartungen des Unternehmensgründers bzw. an den gründungsspezifischen Problemstellungen auszurichten ist. Der Verfasser dieses Beitrages bietet hierbei im Rahmen eines Partner-Netzwerkes eine umfassende Unterstiitzung an, die sowohl als Beratungsleistung (Coaching) als auch in seminaristischer Form erfolgen kann.

\section{Literaturverzeichnis}

Dieterle, W. / Winckler, E.: Gründungsplanung und Gründungsfinanzierung, München 1995

Dilts, R.: Roots of Neuro-Linguistic Programming, Cupertino 1983

Dilts, R.: Walt Disney, Ben Lomond 1990

Gerken, G.: Trend-Zeit, Düsseldorf 1993

Kluczny, J.W.: Die „als-ob“ Technik, in: MultiMind - NLP aktuell, Letter 4/5 1992, S. 23 ff.

Kluczny, J.W.: NLP Practitioner Training. Manual, Berlin 1992

Schnappauf, R.A.: NLP - Die Magie der 5 Sinne, in: Manager Seminare, Heft 3 1991, S. 22 ff.

\section{Fußnoten}

1 vgl. Dilts (1983)

2 vgl. Schnappauf (1991)

3 vgl. Schnappauf (1991) 40

4 vgl. Kluczny (1992)

5 vgl. Kluczny (1992)

6 Gerken (1993) 12

7 vgl. hierzu auch Dilts (1990), der die Entwicklungsmuster Disneys modelliert hat

8 vgl. Kluczny (1992) $23 \mathrm{ff}$.

9 vgl. hierzu auch Dieterle/Winckler (1995)

\section{Verfasser}

Prof. Dr. Willi K.M. Dieterle

Technische Fachhochschule Wildau

Fachbereich Wirtschaft, Verwaltung und Recht

Tel. (03375) 508-953 